\title{
Partial dynamical symmetry and the vibrational structure of $\mathrm{Cd}$ isotopes
}

\author{
A. Leviatan ${ }^{1, *}, N$. Gavrielov ${ }^{1, * *}$, J.E. García-Ramos ${ }^{2, * * *}$, and $P$. Van Isacker $^{3, * * * *}$ \\ ${ }^{1}$ Racah Institute of Physics, The Hebrew University, Jerusalem 91904, Israel \\ ${ }^{2}$ Departamento de Ciencias Integradas, Universidad de Huelva, 21071 Huelva, Spain \\ ${ }^{3}$ Grand Accélérateur National d'Ions Lourds, CEA/DRF-CNRS/IN2P3, Bvd Henri Becquerel, B.P. 55027, F-14076 Caen, France
}

\begin{abstract}
The recently reported deviations of selected non-yrast states in ${ }^{110} \mathrm{Cd}$ from the expected sphericalvibrator behaviour, is addressed by means of an Hamiltonian with U(5) partial dynamical symmetry. The latter preserves the U(5) symmetry in a segment of the spectrum and breaks it in other states. The effect of intruder states is treated in the framework of the interacting boson model with configuration mixing.
\end{abstract}

The Cd isotopes have been traditionally considered to be a prime example of spherical vibrators. Recently, advanced experimental studies have reported significant deviations from this behaviour in selected two- and threephonon states, along the $\mathrm{Cd}$ chain $(\mathrm{A}=108-126)$ [1-3]. These observations have led to claims for the "breakdown of the vibrational motion" in these isotopes and the need for a paradigm shift $[1,2]$. In the present contribution, we examine an alternative explanation for the structure of the Cd isotopes, in terms of $\mathrm{U}(5)$ partial dynamical symmetry (PDS) [4].

A convenient starting point for describing spherical nuclei is the $\mathrm{U}(5)$ limit of the interacting boson model (IBM) [5], corresponding to the chain of nested algebras,

$$
\mathrm{U}(6) \supset \mathrm{U}(5) \supset \mathrm{SO}(5) \supset \mathrm{SO}(3) \text {. }
$$

The basis states $\left|[N], n_{d}, \tau, n_{\Delta}, L\right\rangle$ have quantum numbers which are the labels of irreducible representations of the algebras in the chain. Here $N$ is the total number of monopole $(s)$ and quadrupole $(d)$ bosons, $n_{d}$ and $\tau$ are the $d$-boson number and seniority, respectively, and $L$ is the angular momentum. The multiplicity label $n_{\Delta}$ counts the maximum number of $d$-boson triplets coupled to $L=0$. The dynamical symmetry (DS) Hamiltonian has the form

$$
\hat{H}_{\mathrm{DS}}=t_{1} \hat{n}_{d}+t_{2} \hat{n}_{d}^{2}+t_{3} \hat{C}_{\mathrm{SO}(5)}+t_{4} \hat{C}_{\mathrm{SO}(3)},
$$

where $\hat{C}_{\mathrm{G}}$ is the Casimir operator of $\mathrm{G}$, and $\hat{n}_{d}=$ $\sum_{m} d_{m}^{\dagger} d_{m}=\hat{C}_{\mathrm{U}(5)} . \hat{H}_{\mathrm{DS}}$ is completely solvable with eigenstates $\left|[N], n_{d}, \tau, n_{\Delta}, L\right\rangle$ and eigen-energies

$$
E_{\mathrm{DS}}=t_{1} n_{d}+t_{2} n_{d}^{2}+t_{3} \tau(\tau+3)+t_{4} L(L+1) .
$$

A typical U(5)-DS spectrum exhibits $n_{d}$-multiplets of a spherical vibrator, with a two-phonon $\left(n_{d}=2\right)$ triplet of states $(L=4,2,0)$ at an energy $E\left(n_{d}=2\right) \approx 2 E\left(n_{d}=1\right)$

\footnotetext{
*e-mail: ami@phys.huji.ac.il

**e-mail: noam.gavrielov@mail.huji.ac.il

***e-mail: enrique.ramos@dfaie.uhu.es

****e-mail: isacker@ganil.fr
}

above the ground state $\left(n_{d}=L=0\right)$, and a three-phonon $\left(n_{d}=3\right)$ quintuplet of states $(L=6,4,3,0,2)$ at $E\left(n_{d}=3\right) \approx$ $3 E\left(n_{d}=1\right)$. A quadrupole operator proportional to

$$
\hat{Q}=d^{\dagger} s+s^{\dagger} \tilde{d}
$$

enforces strong $\left(n_{d}+1 \rightarrow n_{d}\right)$ E2 transitions with particular ratios, e.g., $\frac{B\left(E 2 ; n_{d}=2, L=0,2,4 \rightarrow n_{d}=1, L=2\right)}{B\left(E 2 ; n_{d}=1, L=2 \rightarrow n_{d}=0, L=0\right)}=2 \frac{(N-1)}{N}$.

The empirical spectrum of ${ }^{110} \mathrm{Cd}$ consists of both normal levels [shown in Fig. 1(a)], and intruder levels [shown in Fig. 2(a)] based on 2p-4h proton excitations across the $\mathrm{Z}=50$ closed shell. The experimental energies and $\mathrm{E} 2$ rates in Fig. 1(a), demonstrate that most normal states have good spherical vibrator properties, and conform well with the U(5)-DS calculation shown in Fig. 1(b). However, the measured rates for E2 decays from the non-yrast states, $0_{3}^{+},\left(n_{d}=2\right)$ and $\left[0_{4}^{+}, 2_{5}^{+}\left(n_{d}=3\right)\right]$, reveal marked deviations from this behaviour. In particular, $B\left(E 2 ; 0_{3}^{+} \rightarrow\right.$ $\left.2_{1}^{+}\right)<7.9, B\left(E 2 ; 2_{5}^{+} \rightarrow 4_{1}^{+}\right)<5, B\left(E 2 ; 2_{5}^{+} \rightarrow 2_{2}^{+}\right)<0.7_{-0.6}^{+0.5}$ W.u., are extremely small compared to the U(5)-DS values: 46.29, 11.02, 19.84 W.u., respectively. Absolute $\mathrm{B}(\mathrm{E} 2)$ values for transitions from the $0_{4}^{+}$state are not known, but its branching ratio to $2_{2}^{+}$is small.

Attempts to explain the above deviations in terms of strong mixing between the normal spherical [U(5)-like] states and intruder deformed [SO(6)-like] states have been shown to be unsatisfactory $[1,2]$. The reasons are twofold. (i) The strong mixing required for an adequate description of the two-phonon $0_{3}^{+}$state, results in serious disagreements with the observed decay pattern of threephonon yrast states. (ii) The discrepancy in the decays of the non-yrast two- and three-phonon states persists also in the heavier ${ }^{\mathrm{A}} \mathrm{Cd}$ isotopes $(\mathrm{A}=110-126)$, even though the energy of intruder states rises away from neutron midshell, and the mixing is reduced. These observations have led to the conclusion that the normal-intruder strongmixing scenario needs to be rejected, and have raised serious questions on the validity of the multi-phonon interpretation $[1,2]$. In what follows, we consider a possible 

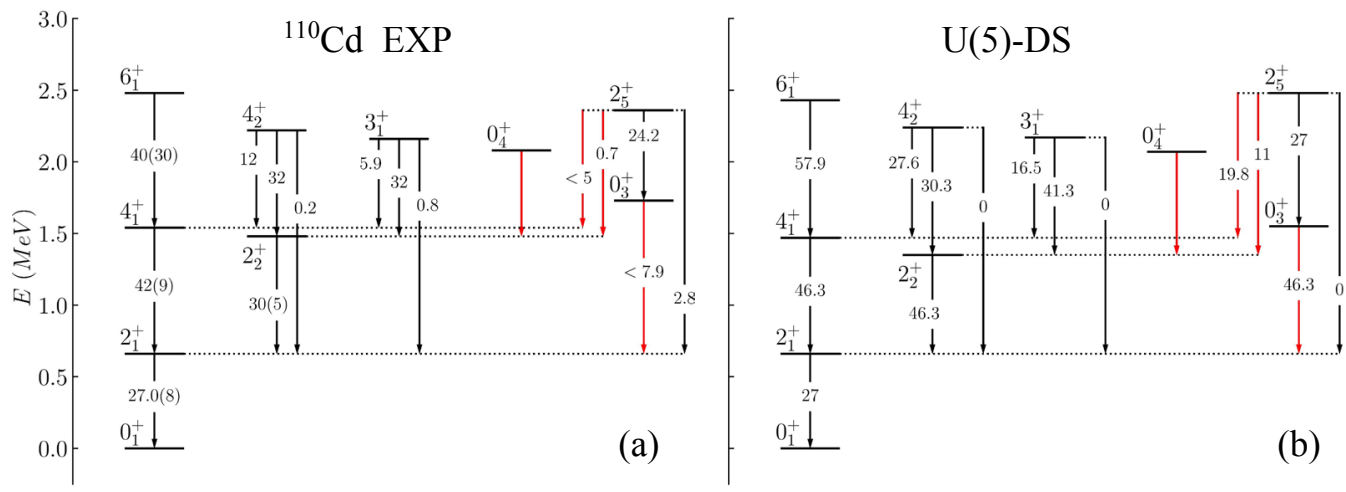

Figure 1. (a) Experimental spectrum and representative E2 rates $[2,6]$ (in W.u.) of normal levels in ${ }^{110} \mathrm{Cd}$. (b) U(5)-DS spectrum obtained from $\hat{H}_{\mathrm{DS}}(2)$ with parameters $t_{1}=641.5, t_{2}=67.9, t_{3}=-26.1, t_{4}=8.7 \mathrm{keV}$ and $N=7$. The E2 operator is $e_{B} \hat{Q}$, Eq. (4), with $e_{B}=1.96$ (W.u. $)^{1 / 2}$. For additional experimental error bars on B(E2) values, not shown in Figs. 1(a) and 2 (a), see [2, 6].
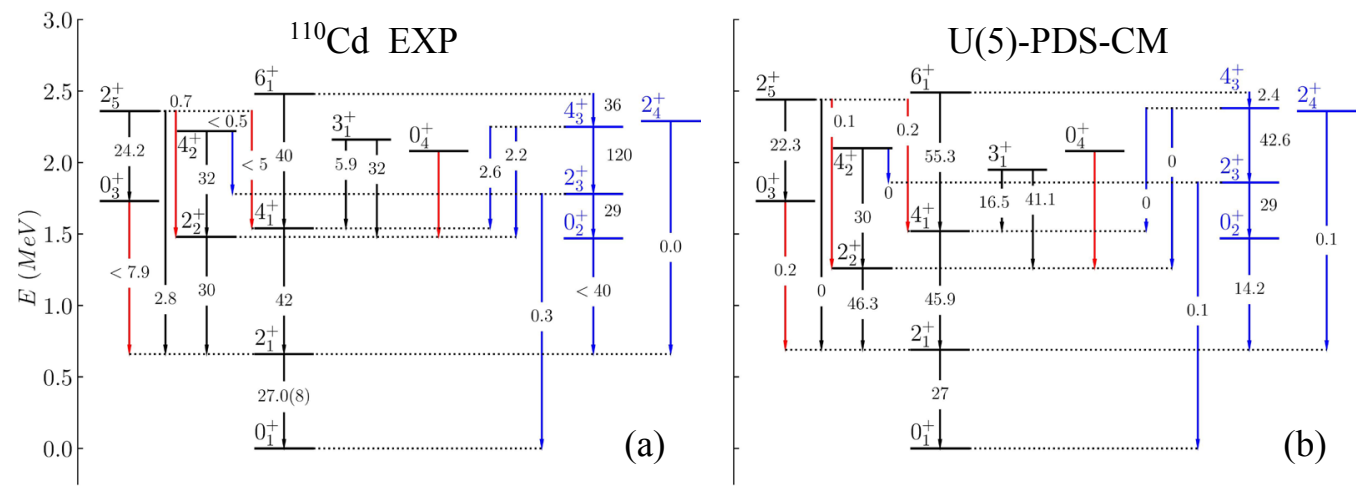

Figure 2. (a) Experimental spectrum and representative E2 rates [2, 6] (in W.u.) of normal [as in Fig. 1(a)] and intruder levels $\left(0_{2}^{+}, 2_{3}^{+}, 4_{3}^{+}, 2_{4}^{+}\right)$in ${ }^{110} \mathrm{Cd}$. (b) U(5)-PDS-CM spectrum, obtained from $\hat{H}$ (11) with parameters $t_{1}=767.8, t_{2}=-t_{3}=73.6, t_{4}=18.5, r_{0}=$ 2.1, $e_{0}=-6.9, \kappa=-72.7, \Delta / 2=4989.5, \alpha=-42.8 \mathrm{keV}$ and $N=7$. The E2 operator (12) used has $e_{B}^{(N)}=1.96, e_{B}^{(N+2)}=1.19$ (W.u. $)^{1 / 2}$.

explanation for the "Cd problem", based on a partial dynamical symmetry (PDS). The latter corresponds to a situation in which a given symmetry is obeyed by only a subset of states and is broken in other states. Such a notion has been previously employed in nuclear spectroscopy in conjunction with the SU(3)-DS [7-9] and SO(6)-DS [10-12] chains of the IBM. In the present contribution, we show the relevance of U(5)-PDS to the Cd problem.

The lowest normal levels comprise three classes of states,

$$
\begin{array}{lll}
\text { Class A : } & n_{d}=\tau=0,1,2,3 & \left(n_{\Delta}=0\right), \\
\text { Class B : } & n_{d}=\tau+2=2,3 & \left(n_{\Delta}=0\right), \\
\text { Class C : } & n_{d}=\tau=3 & \left(n_{\Delta}=1\right) .
\end{array}
$$

In the U(5)-DS calculation of Fig 1(b), the "problematic" states $\left[0_{3}^{+}\left(n_{d}=2\right)\right.$ and $\left.2_{5}^{+}\left(n_{d}=3\right)\right]$ belong to class $\mathrm{B}$, and $0_{4}^{+}\left(n_{d}=3\right)$ belongs to Class $\mathrm{C}$. The remaining "good" spherical-vibrator states $\left[0_{1}^{+}\left(n_{d}=0\right) ; 2_{1}^{+}\left(n_{d}=\right.\right.$ $\left.1) ; 4_{1}^{+}, 2_{2}^{+}\left(n_{d}=2\right) ; 6_{1}^{+}, 4_{2}^{+}, 3_{1}^{+}\left(n_{d}=3\right)\right]$ belong to Class A. As mentioned, the spherical-vibrator interpretation is valid for most states in Fig. 1(a), but not all. We are thus confronted with a situation in which some states in the spectrum (assigned to Class A) obey the predictions of $\mathrm{U}(5)$ DS, while other states (assigned to Classes B and C) do not. These empirical findings signal the presence of a partial dynamical symmetry, U(5)-PDS.

The construction of Hamiltonians with U(5)-PDS follows the general algorithm [12,13], by identifying operators which annihilate particular sets of $\mathrm{U}(5)$ basis states. In the present case, we consider the following interaction,

$$
\hat{V}_{0}=r_{0} G_{0}^{\dagger} G_{0}+e_{0}\left(G_{0}^{\dagger} K_{0}+K_{0}^{\dagger} G_{0}\right),
$$

where $G_{0}^{\dagger}=\left[\left(d^{\dagger} d^{\dagger}\right)^{(2)} d^{\dagger}\right]^{(0)}, K_{0}^{\dagger}=s^{\dagger}\left(d^{\dagger} d^{\dagger}\right)^{(0)}$ and standard notation of angular momentum coupling is used. $\hat{V}_{0}$ of Eq. (6) is in normal-ordered form and satisfies

$$
\hat{V}_{0}\left|[N], n_{d}=\tau, \tau, n_{\Delta}=0, L\right\rangle=0,
$$


with $L=\tau, \tau+1, \ldots, 2 \tau-2,2 \tau$. Eq. (7) follows from the fact that the indicated states have $n_{d}=\tau$ and $n_{\Delta}=0$, hence do not contain a pair or a triplet of $d$-bosons coupled to $L=0$ and, as such, are annihilated by $K_{0}$ [5] and $G_{0}$ [14].

The states of Eq. (7), which include those of Class A, form a subset of $U(5)$ basis states, hence remain solvable eigenstates of the following Hamiltonian

$$
\hat{H}_{\mathrm{PDS}}=\hat{H}_{\mathrm{DS}}+\hat{V}_{0},
$$

with good U(5) symmetry and energies given in Eq. (3) with $n_{d}=\tau$. It should be noted that while $\hat{H}_{\mathrm{DS}}(2)$ is diagonal in the U(5)-DS chain (1), the $r_{0}$-term $\left(e_{0}\right.$-term) in $\hat{V}_{0}$ connects states with $\Delta n_{d}=0$ and $\Delta \tau=0, \pm 2, \pm 4, \pm 6$ $\left(\Delta n_{d}= \pm 1\right.$ and $\Delta \tau= \pm 1, \pm 3$ ). Accordingly, the remaining eigenstates of $\hat{H}_{\text {PDS }}(8)$, in particular those of classes B and $\mathrm{C}$, are mixed with respect to $\mathrm{U}(5)$ and $\mathrm{SO}(5)$. The $\mathrm{U}(5)$ DS is therefore preserved in a subset of eigenstates but is broken in other states. By definition, $\hat{H}_{\text {PDS }}$ exhibits U(5)PDS. Cubic terms of the type present in $\hat{V}_{0}$, Eq. (6), are frequently encountered in PDS Hamiltonians, e.g., in conjunction with signature splitting [9], band structure [10$12]$, and shape-coexistence $[15,16]$ in nuclei.

The effect of intruder levels can be studied in the framework of the interacting boson model with configuration mixing (IBM-CM) [17]. The latter involves the space of normal states described by a system of $N$ bosons representing valence nucleon pairs, and the space of intruder states described by a system of $N+2$ bosons, accounting for particle-hole shell model excitations. This procedure has been used extensively in describing coexistence phenomena in nuclei [18-21]. In the present study of ${ }^{110} \mathrm{Cd}$, the Hamiltonian in the normal sector is taken to be $\hat{H}_{\text {PDS }}$ of Eq. (8), acting in a space of $N=7$ bosons. The Hamiltonian in the intruder sector is taken to be of SO(6)-type [18],

$$
\hat{H}_{\text {intrud }}=\kappa \hat{Q} \cdot \hat{Q}+\Delta,
$$

acting in a space of $N=9$ bosons, with $\hat{Q}$ given in Eq. (4). A mixing term between the $[N]$ and $[N+2]$ boson spaces is defined as [18-21],

$$
\hat{V}_{\text {mix }}=\alpha\left[\left(s^{\dagger}\right)^{2}+\left(d^{\dagger} d^{\dagger}\right)^{(0)}\right]+\text { H.c, }
$$

where H.c. means Hermitian conjugate. The combined Hamiltonian for the two configurations has the form

$$
\hat{H}=\hat{H}_{\mathrm{PDS}}^{(N)}+\hat{H}_{\text {intrud }}^{(N+2)}+\hat{V}_{\text {mix }}^{(N, N+2)} .
$$

Here $\hat{O}^{(N)}=\hat{P}_{N}^{\dagger} \hat{O} \hat{P}_{N}$ and $\hat{O}^{\left(N, N^{\prime}\right)}=\hat{P}_{N}^{\dagger} \hat{O} \hat{P}_{N^{\prime}}$ for an operator $\hat{O}$, with $\hat{P}_{N}$ a projection operator onto the $[N]$ boson space. Similarly, the E2 operator is defined as,

$$
\hat{T}(E 2)=e_{B}^{(N)} \hat{Q}^{(N)}+e_{B}^{(N+2)} \hat{Q}^{(N+2),}
$$

with boson effective charges, $e_{B}^{(N)}$ and $e_{B}^{(N+2)}$.

The experimental energies and E2 rates for both normal and intruder levels in ${ }^{110} \mathrm{Cd}$, are shown in Fig. 2(a). They are well reproduced by an IBM-PDS-CM calculation, shown in Fig. 2(b), employing the Hamiltonian of Eq. (11) and the E2 operator of Eq. (12). The mixing between the intruder states and normal states of class A is weak. The latter states retain a high degree of purity and good U(5) quantum numbers. This is reflected in their E2 decay properties, which are essentially the same as those of the U(5)-DS shown in Fig. 1(b). In contrast, the states in classes $\mathrm{B}$ and $\mathrm{C}$, whose decay properties show marked deviations from the U(5)-DS limit, are mixed with other normal and intruder states. The resulting calculated values: $B\left(E 2 ; 0_{3}^{+} \rightarrow 2_{1}^{+}\right)=0.25, B\left(E 2 ; 2_{5}^{+} \rightarrow 4_{1}^{+}\right)=0.19$, $B\left(E 2 ; 2_{5}^{+} \rightarrow 2_{2}^{+}\right)=0.12$ W.u., are consistent with the measured upper limits: $7.9,5,0.7_{-0.6}^{+0.5}$ W.u., respectively.

In summary, we have considered the vibrational structure of ${ }^{110} \mathrm{Cd}$, by means of U(5)-PDS. The PDS Hamiltonian retains good $\mathrm{U}(5)$ symmetry for yrast states, but breaks it in particular non-yrast states. The mixing with the intruder levels is weak, and affects mainly the broken U(5)-DS states. Most low-lying normal states maintain the vibrational character and only specific states exhibit a departure from this behaviour, in line with the empirical data. Calculations are underway to see if this approach can be implemented in other neutron-rich $\mathrm{Cd}$ isotopes.

This work is supported in part (A.L. and N.G.) by the Israel Science Foundation (Grant 586/16).

\section{References}

[1] P.E. Garrett, K.L. Green and J.L. Wood, Phys. Rev. C 78, 044307 (2008)

[2] P.E. Garrett et al., Phys. Rev. C 86, 044304 (2012)

[3] J.C. Batchelder et al., Phys. Rev. C 89, 054321 (2014)

[4] A. Leviatan, Prog. Part. Nucl. Phys. 66, 93 (2011)

[5] F. Iachello and A. Arima, The Interacting Boson Model (Cambridge Univ. Press, Cambridge, 1987)

[6] G. Gürdal and F.G. Kondev, NDS 113, 1315 (2012)

[7] A. Leviatan, Phys. Rev. Lett. 77, 818 (1996)

[8] A. Leviatan et al., Phys. Rev. C 60, 061301(R) (1999)

[9] A. Leviatan, J.E. García-Ramos and P. Van Isacker, Phys. Rev. C 87, 021302(R) (2013)

[10] P. Van Isacker, Phys. Rev. Lett. 83, 4269 (1999)

[11] A. Leviatan and P. Van Isacker, Phys. Rev. Lett. 89, 222501 (2002)

[12] J.E. García-Ramos, A. Leviatan and P. Van Isacker, Phys. Rev. Lett. 102, 112502 (2009)

[13] Y. Alhassid and A. Leviatan J. Phys. A 25, L1265 (1992)

[14] I. Talmi, in Symmetries in Nuclear Structure, (A. Vitturi and R.F. Casten Eds.), World Scientific p. 10 (2004)

[15] A. Leviatan and D. Shapira, Phys. Rev. C 93, 051302(R) (2016)

[16] A. Leviatan and N. Gavrielov, Phys. Scr. 92, 114005 (2017)

[17] P.D. Duval and B.R. Barrett, Nucl. Phys. A 376, 213 (1982)

[18] K. Heyde et al., Nucl. Phys. A 586, 1 (1995)

[19] R. Fossion, K. Heyde, G. Thiamova and P. Van Isacker, Phys. Rev. C 67, 024306 (2003)

[20] J.E. García-Ramos, V. Hellemans and K. Heyde, Phys. Rev. C 84, 014331 (2011)

[21] J.E. García-Ramos and K. Heyde, Phys. Rev. C 89, 014306 (2014); Phys. Rev. C 92, 034309 (2015) 